\title{
Mechanism of Spectral Distortion for Real-Time Crack Propagation on Aluminum Alloy Structure
}

\author{
Bo Jin $\mathbb{D}^{1},{ }^{1}$ Cunbo Lu, ${ }^{1}$ Feng Zhang, ${ }^{1}$ and Weifang Zhang ${ }^{2}$ \\ ${ }^{1}$ Information Science Academy China Electronics Technology Group Corporation, Building No. 4, 36 Sidaokou North Rd., \\ Haidian Dist., Beijing 100086, China \\ ${ }^{2}$ School of Reliability and Systems Engineering, Beihang University, 37 Xueyuan Rd., Haidian Dist., Beijing 100191, China
}

Correspondence should be addressed to Bo Jin; 403115059@qq.com

Received 21 September 2021; Revised 7 December 2021; Accepted 11 December 2021; Published 7 January 2022

Academic Editor: Haidong Shao

Copyright (c) 2022 Bo Jin et al. This is an open access article distributed under the Creative Commons Attribution License, which permits unrestricted use, distribution, and reproduction in any medium, provided the original work is properly cited.

This study investigates four characteristic damage mechanisms of fiber Bragg grating (FBG) sensors, with fatigue crack propagation in aluminum alloy. The multipeak wavelength distinguish algorithm was developed for FBG spectrum quantitative analysis. The results distinguish a subordinate peak skewing significantly, associated with strain patterns along the FBG, corresponding to various crack lengths. For parallel bonded direction grating, the subordinate peak skewing appears at the strain pattern transition region. This is located at the ratio 32\%-34\% of crack length lying in the crack tip. Meanwhile, the four damage characteristics correspond to subordinate peak skewing. When the strain is distributed along the grating, spectral distortion occurs. In this region, the cubic strain pattern determines the shorter wavelength location of subordinate peaks. This corresponds to the $15 \%-17 \%$ ratio of crack length lying in the grating, causing spectral oscillations.

\section{Introduction}

Aluminum alloy is extensively employed in aircraft construction, because the constructs will experience structural damage in long-term service. This involves mechanisms such as fatigue, material aging, corrosion, and cracking problems [1]. Furthermore, the structural damage can be identified synchronously in real-time monitoring of aluminum alloy crack propagation [2]. Fiber Bragg grating (FBG) sensors have been acknowledged and used in structural health monitoring (SHM). For example, this is implemented in the concrete structure of bridges, nuclear power stations, and large dams. This is due to the following specific benefits of FBG sensors which include erosion resistance, small size, and multiplexing [3]. It is deliberated to be one of the most hopeful sensors in observing the strain distribution near the crack tip for propagation prediction. However, it is a challenge to utilize the FBG sensors in crack propagation monitoring of aluminum alloy components.

Several researchers have made contributions on interpretations for mechanisms of spectral characteristics, regarding the various damage pattern identifications of composite materials. Takeda et al. place FBG sensors near the delamination to correlate spectral intensity and spread with crack progression [4]. They observe that the delamination starts at the end of the sensor and grows parallel along the longitudinal axis. The related spectrum shifts and splits into two distinct peaks. Childs et al. [5] relate these two peaks to the strain levels along the grating ahead and behind the delamination. They attempt to correlate the intensity ratio of these peaks to the delamination length. Sante [6] embedded a chirped FBG sensor near the bond line of a GFRP single-lap joint and validated the relationship between the disbanded tip position and the wavelength of the shape change. In addition, Ramly et al. [7] undertook research on the monitoring composite delamination areas. These results describe two peaks at lower and higher wavelengths in the reflection spectra, corresponding to the various forms of strain distribution. This continues with an increment of the delaminated area.

The shapes of the reflected spectra depend on the strain gradients along the axial grating of the FBG sensors 


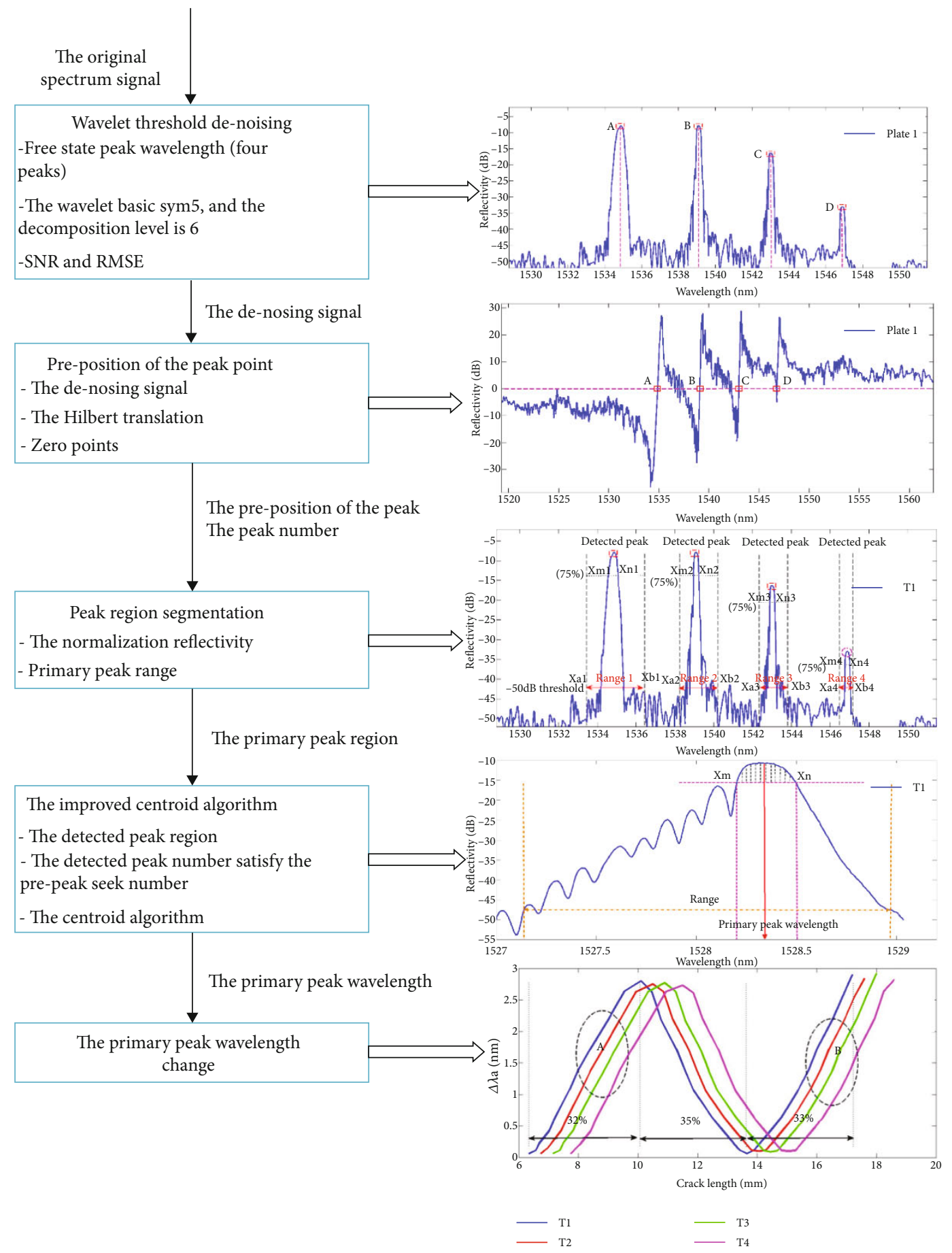

FIGURE 1: The diagram of the centroid peak shifting detection algorithm.

[8]. Thus, Rajabzadeh et al. [8] assume that the nonuniform strain distribution along the grating may be the main reason for spectrum distortion. In addition, numerical models are constructed to verify Rajabzadeh's statement. However, for real-time applications, the spectral distortion (or the subordinate peak appearance time) cannot be used as the determinate factor for crack length propagation. Our research suggests that the skewing of the subordinate peak corresponds to the crack propagation. In addition, Correia et al. [9] found that the turning of the subordinate peak is correlated with the ratio of effective sensing length to the actual bonded length. Also, the sensing grating regions can be divided into three sections called the effective interaction subregions. To analyze the comprehensive correlation of crack length with FBG spectral behavior, we will investigate four damage characteristics. This involves the primary wavelength variation, the number of subordinate peaks, the spectral bandwidth, and the subordinate wavelength skewing, in correspondence with the crack length. 


\section{Damage Characteristic Detection Algorithm}

With the nonuniform strain sensing, the spectrum became asymmetric, broadened, and distorted; the traditional algorithm for extracting the FBG spectral central peak is difficult to realize the accurate wavelength value. In this paper, a multipeak wavelength distinguish algorithm is introduced.

It is necessary to denoise the spectrum signal, and the variation mode decomposition (VMD) combined with wavelet thresholding is developed. The subordinate wavelength data were extracted under the $3 \mathrm{~dB}$ bandwidth region, and the centroid algorithm is defined based on the damage spectrum. The diagram of the centroid peak shifting detection algorithm is shown in Figure 1.

2.1. VMD Wavelet Denoising Pretreatment Algorithm. Traditionally, the variation mode decomposition (VMD) can sense multiple modes concurrently; under the same frequency [10], Li and Yao [11] introduced the band-limited intrinsic mode function (BLIMF) for signal acquisition. However, the algorithm lacks adaptability due to the fixed energy distribution of signal mode, corresponding to the determined decomposition layers. In this paper, a variation mode decomposition integrated with the wavelet threshold algorithm is produced. And the procedure of VMD wavelet denoising algorithm is as follows:

Step 1. The spectrum signal in the domain is decomposed by the VMD algorithm into band-limited intrinsic mode function (BLIMF) $u_{k}$ in the frequency domain. And the optimal decomposition layers' number $K$ is set to 6 , and the corresponding optimal balance parameter $\alpha$ is set as 200.

Step 2. By soft thresholding the BLIMF value, the wavelet type is set to sym 5 and the wavelet layer is set to 6 [12]. The improved soft threshold can be set as

$$
\widehat{d}_{l}= \begin{cases}\operatorname{sign}\left(d_{i}\right)\left(\left|d_{i}\right|-\frac{T_{i}}{\exp \left(\left(\left|d_{i}\right| / T\right)-1\right)^{2}}\right), & \left|d_{i}\right| \geq T_{i}, \\ 0, & \left|d_{i}\right|<T_{i}\end{cases}
$$

where $d_{i}$ represents the $i_{\text {th }}$ improved threshold of the BLIMF. $T_{i}$ represents each submodule threshold, and the solution formula is described as follows:

$$
T=\frac{100 \sigma \sqrt{2 E_{i} \lg (N)}}{\lg (j+1)} .
$$

Here, mean square deviation $\sigma$ is estimated by $\sigma=$ $\operatorname{median}\left(\left|c_{i}\right|: i=1,2 \cdots, N\right) / 0.675$. The $i_{\text {th }}$ BLIMF energy $E_{i}$ is estimated as follows:

$$
\widehat{E}_{k}=\frac{E_{1}^{2}}{\beta} \rho^{-k}, \quad k=2,3,4 \cdots .
$$

The shifting iteration process parameters $\beta, \rho$ are set as 0.719 and 2.01 [13], respectively.

Step 2. The multidenoising is applied with various methods, such as the EMD with the detrend fluctuation analysis (EMD-DFA), EMD with the changed thresholding wavelet (EMD-changed wavelet), EMD-wavelet, and VMDchanged wavelet, as shown in Figure 2.

The VMD-changed wavelet method can efficiently preserve the detail signal without affecting the noise signal filtered.

2.2. The Multipeak Wavelength Distinguish Algorithm. The deformed spectrum was split primarily for multipeak prepositioning, and secondarily the spectrum central wavelength was extracted segmentally. The detailed process is shown in Figure 3.

To extract the zero crossing points of the transformed signal, the denoised signal must be transformed by the Hilbert transform firstly. The Hilbert transform value equals the convolution integral between the signal $x(t)$ and $(\pi t)^{-1}$. The Hilbert transform $x(t)$ was denoted as

$$
\widehat{x}(t)=H[x(t)]=x(t) * \frac{1}{\pi t} .
$$

The denoising spectrum signal is shown in Figure 4(a), and the processed signal by the Hilbert transform is shown in Figure 4(b). To reduce the error of extracting extreme poles instead of zero crossing points, the peak slope and peak groupthreshold method is proposed for the initial estimate of the primary peak area. The subsidiary peak recognition satisfy not only the value of zero crossing points under the Hilbert transform but also the peak threshold sharp increased $1 \mathrm{~nm}$ in slop shape, as shown in Figure 4(b). Meanwhile, to improve the accuracy of detection, the $3 \mathrm{~dB}$ threshold window function is defined in the preprocessing, as shown in Figure 4(c).

The intensity of $\widehat{x}(\lambda)$ has a sharp slop between the left and right pole points in Figure 4(b). Additionally, the intensity of $x(\lambda)$ in Figure 4(a) and $\widehat{x}(\lambda)$ in Figure 4(b) have the same fluctuating trend in the reflection area. The subsidiary peak number and the FWHM can be estimated.

Due to the advantage of high computation and precision, the centroid algorithm is applied to recognize the peak wavelength, as shown in Figure 5. The centroid algorithm produces a point corresponding to the geometric centroid of a spectrum, calculated by equation (5), where $N$ is the size of the spectrum point vector, $\lambda_{i}$ is the $i_{\text {th }}$ point wavelength, and $I_{i}$ is the $i_{\text {th }}$ point reflectivity intensity.

$$
\lambda_{B}=\frac{\sum_{i=1}^{N} \lambda_{i} I_{i}}{\sum_{i=1}^{N} I_{i}} .
$$

\section{Experiment Performance}

To investigate the mechanisms of damage characteristics of different fatigue crack lengths, the employed specimens are bonded with FBG sensors. In the existing literature, the FBG sensors were embedded in composites for structural health monitoring. Ramakrishnan et al. [14] found that the 


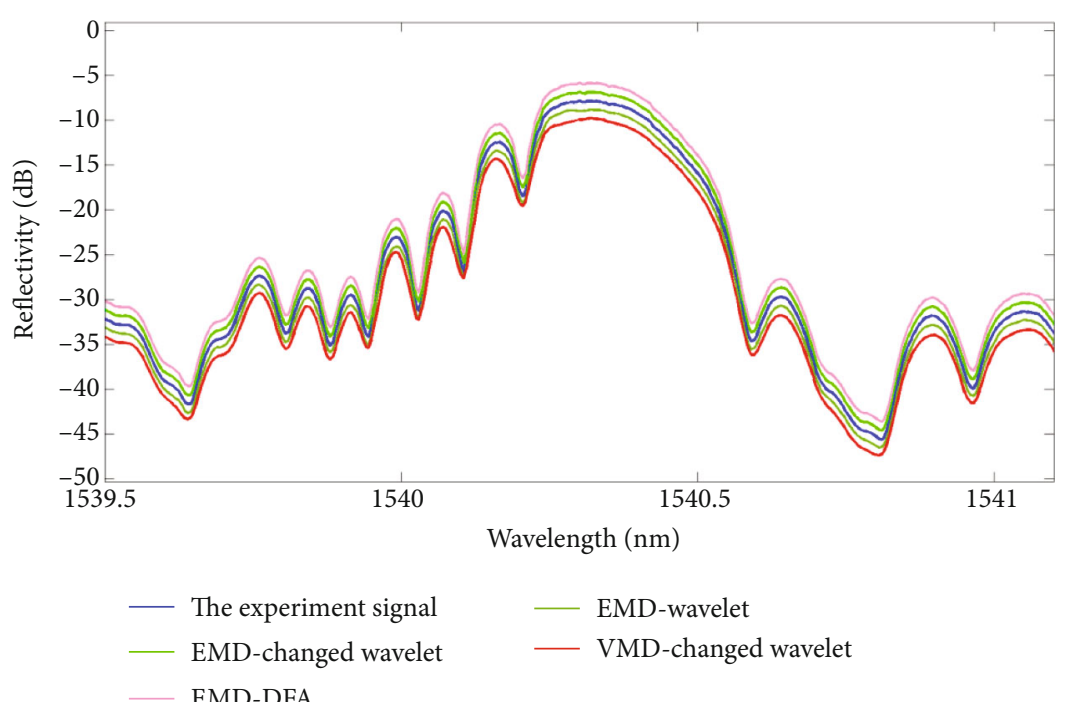

(a)

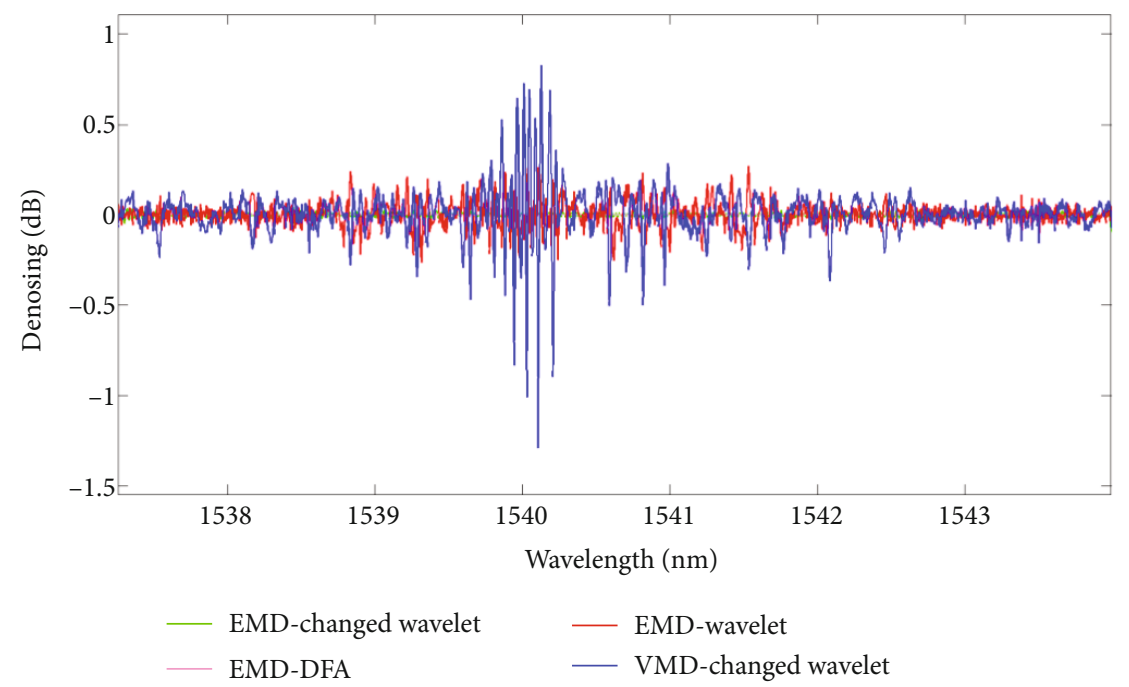

(b)

Figure 2: (a) The original spectrum signal. (b) The denoising signal by the proposed algorithm.

crack length was closely related to the delamination damage of composites, and the FBG sensors could sense the composite delamination. However, it is still a challenge for FBG to sense the crack damage of aluminum alloy. In this paper, the FBG sensors are adhered on the aluminum alloy plate, and the mechanism of damage characteristics corresponding to the crack propagation is studied. In this section, we evaluate the conduct for four proposed damage characteristics on FBG spectral experimental data. Therefore, an experiment in real-time crack propagation monitoring was performed with a high-resolution optical microscope SM125. In addition, the reflectance spectra were obtained and recorded with an optical spectral analyzer.

Strain field alternation caused changes in the full-spectral response that were detected and indicated that disband dependent nonlinear behavior of the structure appears in the dynamic spectral response. Jin et al. [15] found that the subordinate peak skewing corresponds closely to the crack length and location. Meanwhile, further investigation on the subordinate peak skewed various with the ratio of crack length to the entire grating, which indicates that the first appeared time and the skewing of the subordinate peak are indicated by the crack location and length. Consequently, a real-time peak-seek algorithm for subordinate peak detection is applied, aided by the model carol method [16]. To calculate the peak width and subordinate peak location, the algorithm detects the maximum reflected optical power of each FBG.

3.1. Specimen. The objective system established in this paper is a plate made of aluminum alloy 7075-T6, with the dimensions of $300 \mathrm{~mm} \times 100 \mathrm{~mm} \times 2 \mathrm{~mm}$, as shown in Figure 6. A $10 \mathrm{~mm}$ hole is in the center of the plate. To follow the crack tip with the FBG sensors, a $3 \mathrm{~mm}$ initial crack length is desired according to the standard. The specimen properties are shown in Table 1.

For strain sensing in various shape distributions along the grating, the FBG sensors with no covering layer are 


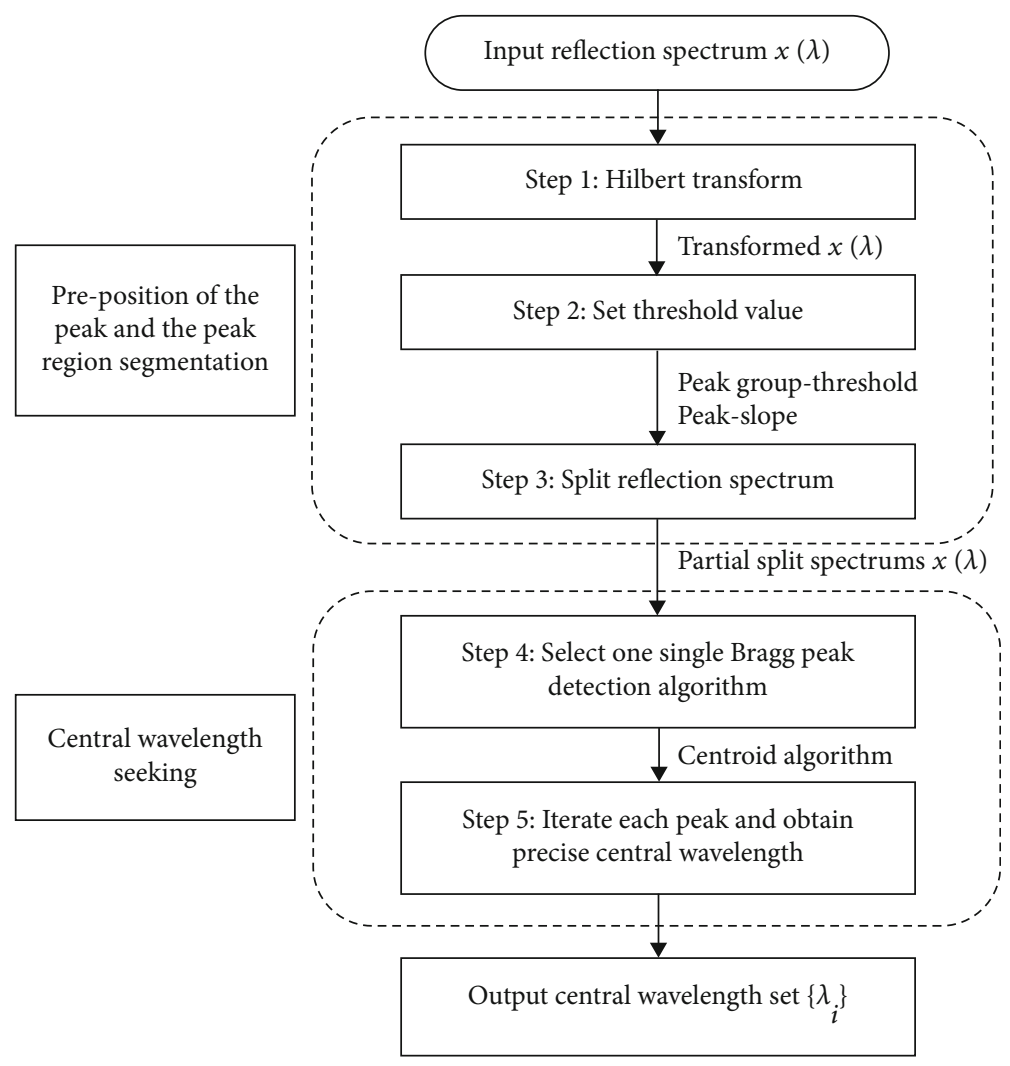

FIgURE 3: The diagram of multipeak central value diagnosis.

directly bonded in a horizontal plane onto the hole side in the 7075-T6 aluminum alloy specimen. This ensures a slow propagation of the crack into the sensing zone, and the FBG1 was placed $4 \mathrm{~mm}$ ahead of the initial crack tip with a $4 \mathrm{~mm}$ distance between FBG1 and FBG2. FBG parameters adopted in the experiment are as follows: grating length is 10.1 and $10.2 \mathrm{~mm}$; bonded length is 11.3 and $11.4 \mathrm{~mm}$; thickness of adhesive layer is about $0.3 \mathrm{~mm}$; the liquid cyanoacrylate adhesive is used; and Young's modulus of the adhesive is $1.75 \mathrm{MPa}$.

\subsection{Experiment Setup. A fatigue crack damage recognition} test platform is developed, and FBG sensors are utilized to abstract the damage indicator. The overall view of the hole-edge crack experimental setup is composed of three main segments: fatigue loading equipment, optical modulation analyzer, and fatigue crack detection device (see Figure 7). Fatigue testing is undertaken using a hydraulic MTS machine with continuous fatigue loading along the axial direction. Constant amplitude loading spectra are used in this study, with a maximum loading set value of $65 \mathrm{MPa}$ and a cycling frequency of $10 \mathrm{~Hz}$. The FBG sensors were connected to an optical demodulator (SM125, Micro Optics Inc.), with high wavelength resolution. The reflected spectral data was recorded for real-time monitoring of crack propagation. Fatigue crack length was measured with an optical microscope, and crack tip propagation was observed with a Charge-Coupled Device (CCD) camera.

3.3. Mechanism of Damage Characteristics. The natural crack was initiated and propagated by submitting the plate to a cyclical fatigue test. An FBG sensor was used to measure the strain distribution along the axial grating at different strain profiles, with the crack propagation. Data was obtained using the FBG sensing demodulation system throughout cyclic fatigue. Initially, the reflection spectra were symmetrical and developed a primary reflection spectrum. Subsequently, the peak simply moves backwards or forwards when the grating sensed a uniform strain profile. In this stage, its pitch changes accordingly with internal periodic modulation.

With the crack propagation, the spectral wavelength shifted gradually while the reflection spectrum showed a significant change in its shape. For example, Figure 8 shows when the subordinate peaks appear. In this period, FBG sensors have a gradual change in the grating period, which yield a characteristically broad and flat reflection spectrum. The subordinate peak skewing of the reflected spectra has a one-to-one correspondence with the proportion of crack length in the grating section. Meanwhile, the subordinate peak number changes with the fatigue crack propagation. During this process, the changing regularity of subordinate peak numbers closely relates to the proportion of crack length in the grating length. Noteworthy meaningful phenomena are that spectral distortion appears in the stage of ratio alternation. The distortion of the spectra is attributed to the eventually nonuniform distribution of strain along the grating.

Figure 8 shows the signal changes of the FBG sensors due to different crack lengths under cyclic crack propagation tests. The above-mentioned features would be applied to identify damage in real-time monitoring. 


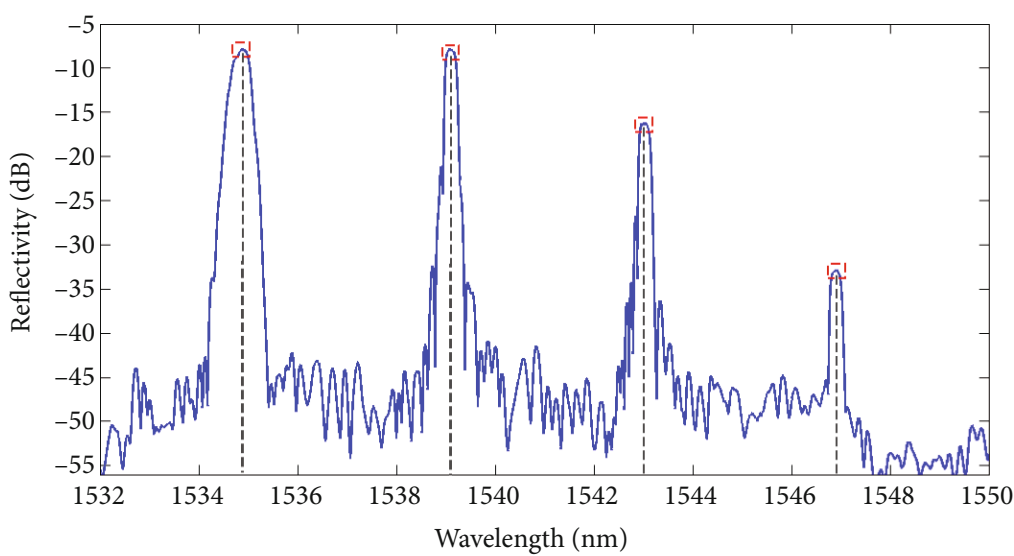

$-\mathrm{T} 1$

(a)

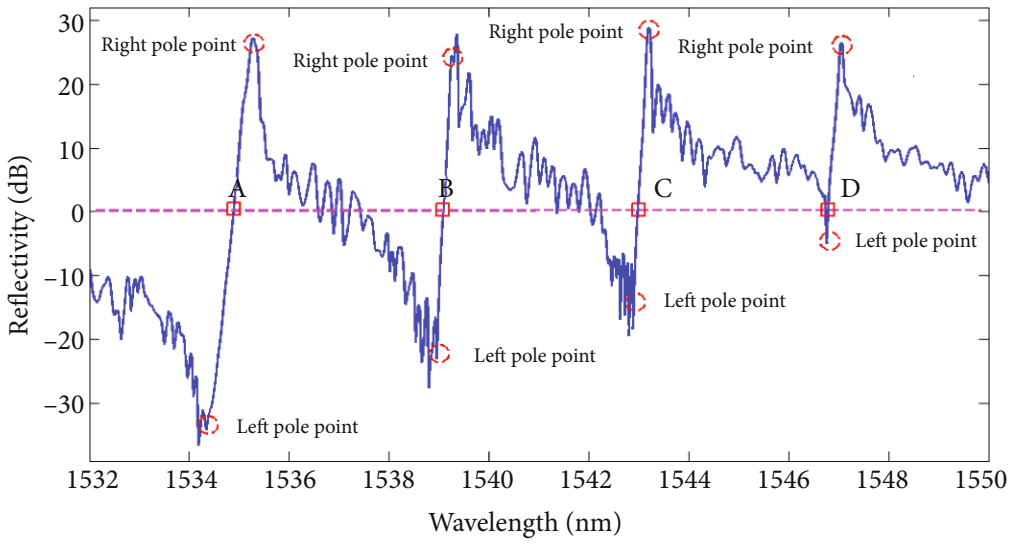

- T1

(b)

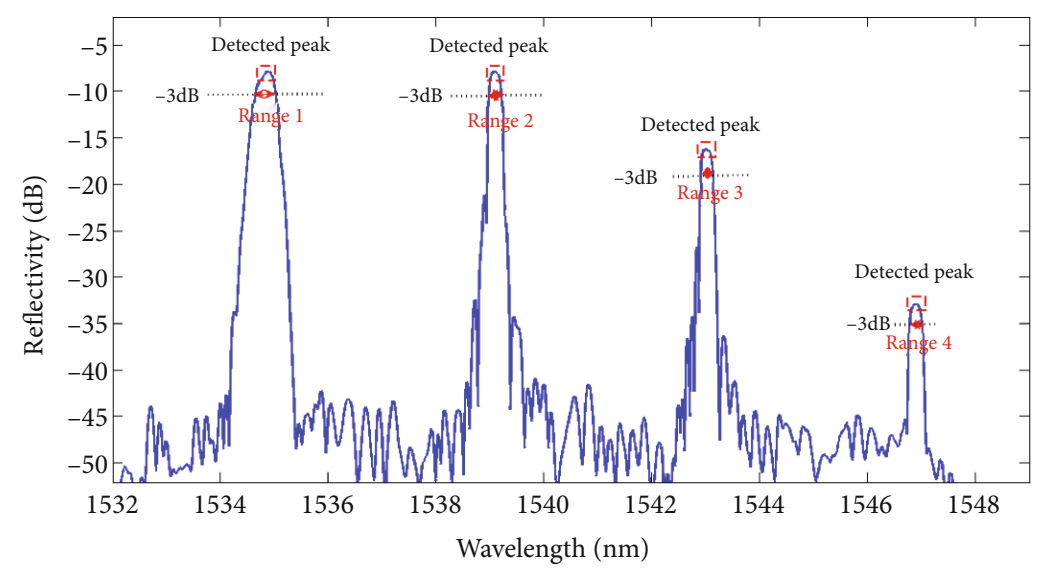

(c)

FIgure 4: (a) The original spectrum. (b) The processed signal treatment by the Hilbert transform. (c) Peak diagnosis using the proposed algorithm.

3.3.1. The Primary Wavelength Variation. It is well understood that the primary wavelength corresponds to the average strain along the grating. When the grating senses compression/strain, the primary wavelength shifts towards shorter/ higher wavelength. Fernando et al. [17] illustrate the primary wavelength corresponding to the delamination with strain magnitude. According to Reference [18], the center wavelength increased linearly with the composite delamination damage. 


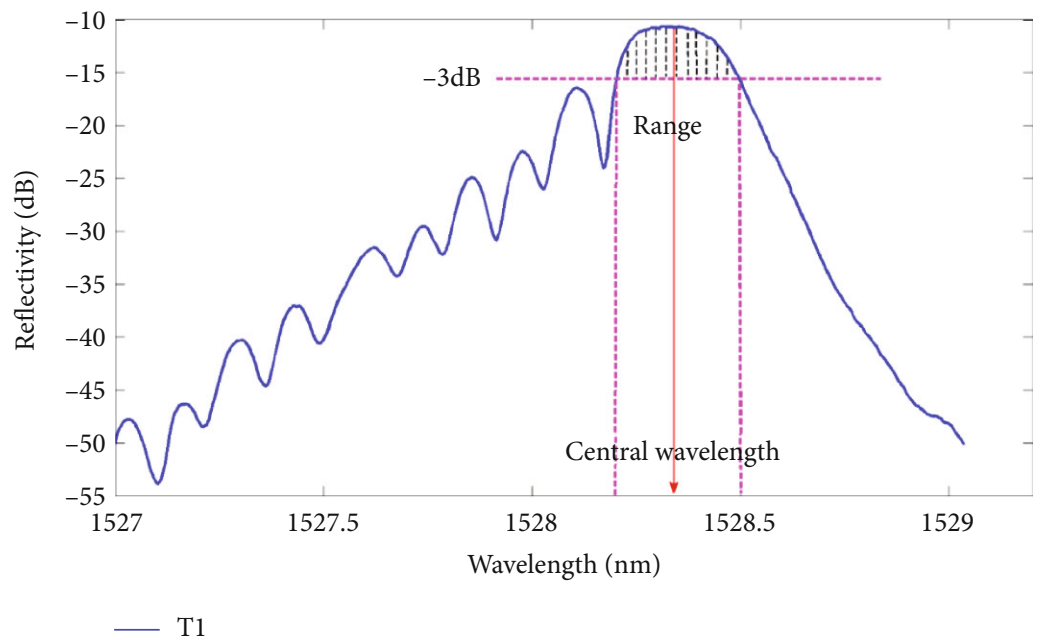

Figure 5: Schematic of the central wavelength detection by the centroid algorithm.

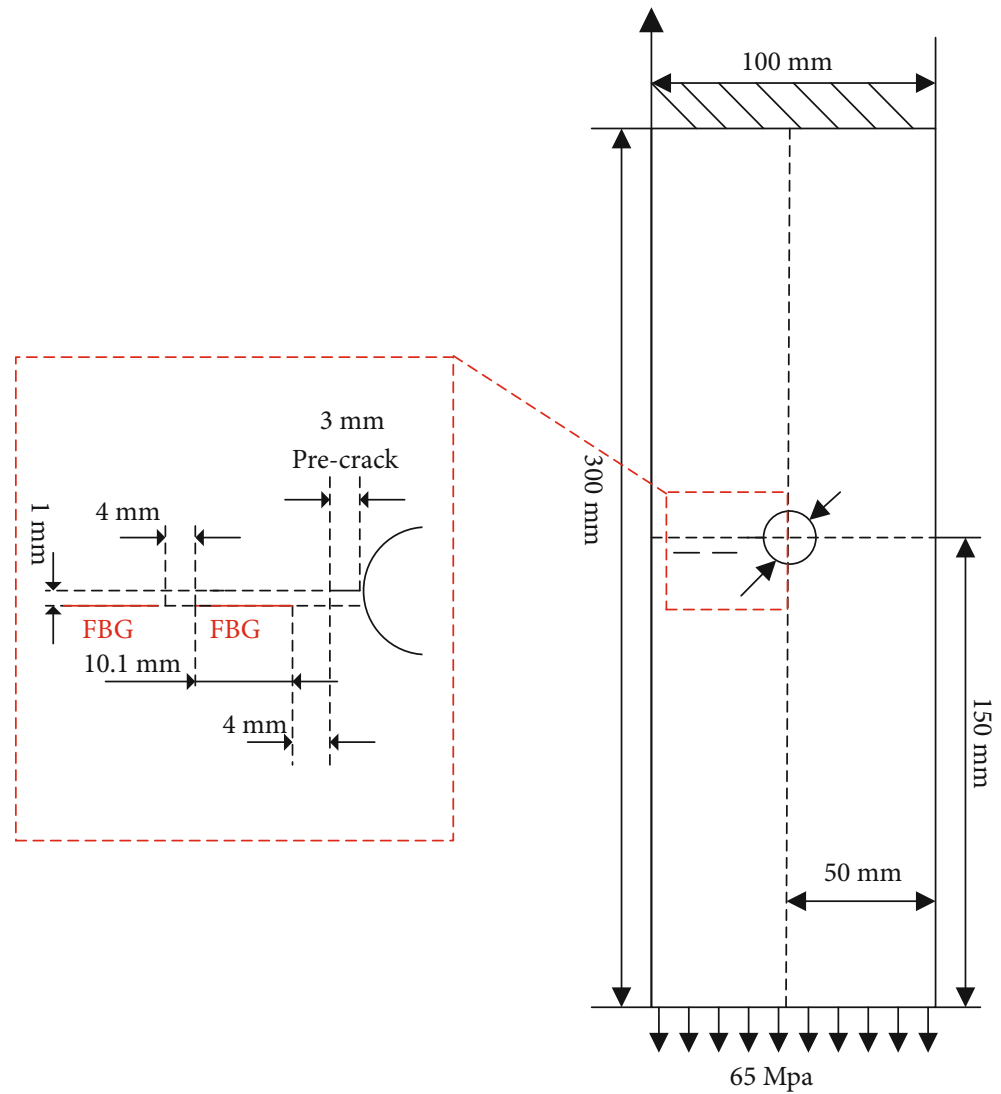

FIGURE 6: Schematic of the aluminum specimen.

TABLE 1: Mechanical properties of 7075-T6 aluminum alloy plates.

\begin{tabular}{lcccc}
\hline Material & $\begin{array}{c}\text { Tensile } \\
\text { strength } \\
(\mathrm{MPa})\end{array}$ & $\begin{array}{c}\text { Yield } \\
\text { strength } \\
(\mathrm{MPa})\end{array}$ & $\begin{array}{c}\text { Poisson's } \\
\text { ratio }(\mathrm{MPa})\end{array}$ & $\begin{array}{c}\text { Elastic } \\
\text { modulus } \\
(\mathrm{MPa})\end{array}$ \\
\hline $\begin{array}{l}\text { AL7075- } \\
\text { T6 }\end{array}$ & 572 & 503 & 0.33 & 73100 \\
\hline
\end{tabular}

However, FBG sensors used for fatigue-induced damage monitoring were mainly bonded perpendicular to the external loading force. However, this research applies a force perpendicular to the fast axis of the fiber, and the crack propagation orientation is paralleled to the fast axis of the fiber. The experimental result shows that the reflection spectra were clearly narrow and symmetrical. Therefore, the primary reflection spectrum is only found at the initial state. 


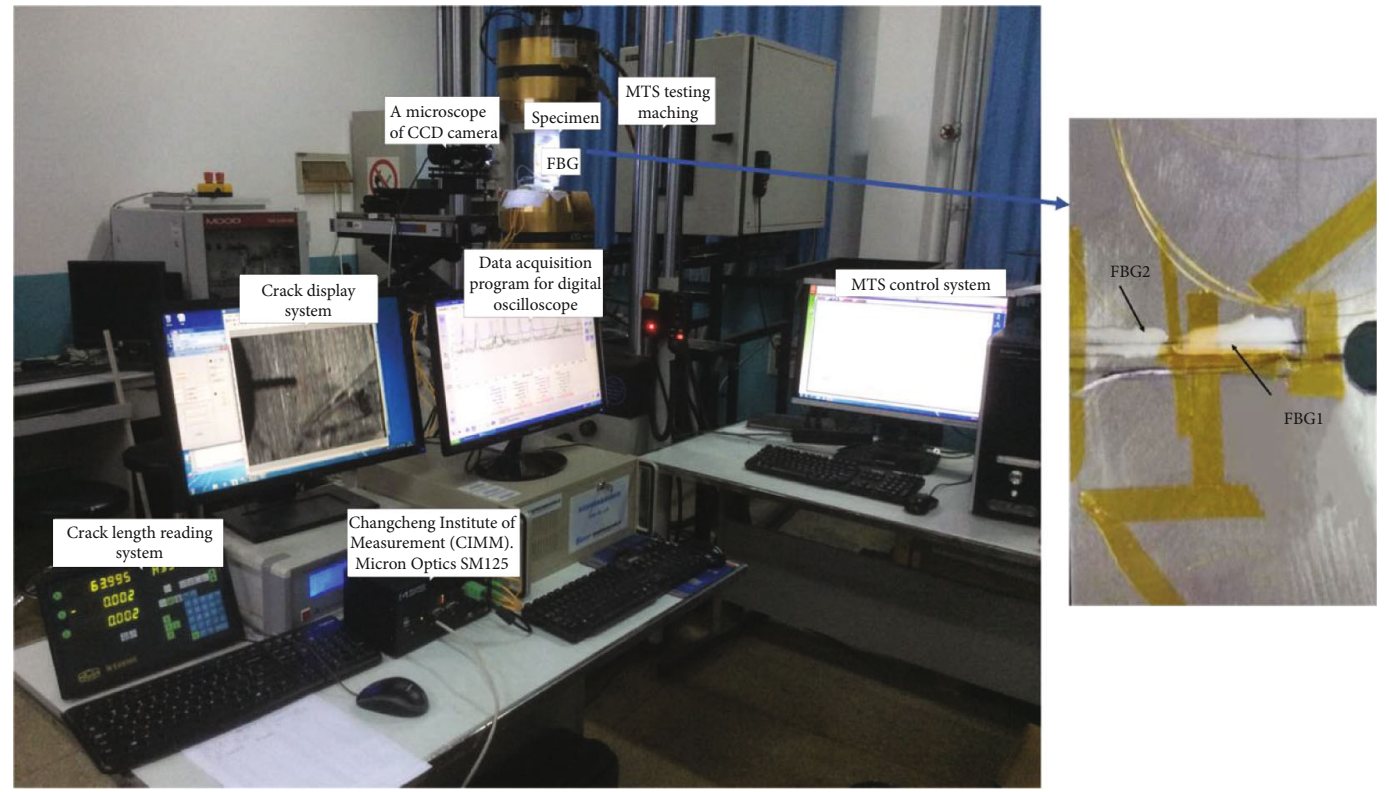

FIGURE 7: Experiment setup for the crack detection in aluminum structure.

Afterwards, the peak simply moves backwards or forwards when the grating sensed a uniform strain profile. In this stage, its pitch changes accordingly with internal periodic modulation. It was also observed that primary wavelength shift value was less than $0.1 \mathrm{~nm}$ during crack propagation. This is because there is a small offset between the center of the FBG and the intersection point between the FBG and neutral line.

With crack propagation, the nonuniform stain distribution is induced by the crack tip causing the reflection spectrum deformation. It should be noted here that the turning point coincides well within the ratio of crack length lying in the whole grating. At the ratio 32\%-35\% of crack tip located on the grating, the average strain value is distributed on the effective sensing. This value is relatively large, and the cubic strain pattern is distributed along the grating taking primary responsibility in spectral peak location decision. Thus, in regions $A$ and $B$, the wavelength increased with crack propagation. When the ratio reaches about $36 \%-67 \%$, the average strain value decreased and the quadratic strain pattern is distributed along the grating. Figure 9 shows the primary wavelength due to different crack lengths.

3.3.2. Spectral Bandwidth. The spectral bandwidth measurements are well represented by the average strain gradient along the FBG sensors. However, this does not yield an accurate measurement of the complexity of the strain field. Indeed, many researchers [19] found that the spectral bandwidth could respond to strain gradient. Hence, it had been applied for real-time in situ monitoring of composite material damage. Ussorio et al. [20] used the spectral bandwidth changes of distributed FBG sensors as indicators of matrix crack. Takeda et al. [21] estimated the composite delamination based on the quantitative change in the spectral bandwidth.
The spectral bandwidth indicator could be applied for both composites and metal materials. Jin et al. [22] have demonstrated that strain distributions along the grating creates localized resonances at different wavelengths by experimental studies. This provides a reason why the spectral distortion occurred. Meanwhile, the number of the subordinate peaks could significantly affect the spectrum bandwidth variation. Thus, spectral bandwidth would be used as an indicator for the hole-edge crack diagnosis. Spectral bandwidth represents the range of the induced strain values and thus the magnitude of the strain gradient along the grating. This research will utilize the full width at half maximum (FWHM). Accordingly, the spectral bandwidth is the most commonly used measurement of spectral distortion for FBG sensors [23].

Previous studies have demonstrated that the strain distribution along the grating is mainly due to the ratio of crack length lying in the grating with respect to the entire grating. Another observation could be made for spectral changes, due to crack propagation in which significant peak splitting occurs. However, it should be noted the total bandwidth increase may not be large in this period. The FWHM variation tendency shows gentle rise and decline in zones $\mathrm{A}$ and $\mathrm{B}$, as shown in Figure 10. Meanwhile, the FWHM displays an obvious correlation with crack length until a crack saturation length was reached. The spectrum bandwidth was also directly related to strain distribution and has been previously applied [24]. This is because there is a small offset between the center of the FBG and the intersection point between the FBG and the neutral line.

It is known that the deformity of the reflection spectra is generally related to the strain distribution along the FBGs [25]. In fact, the distribution of strain patterns along the grating corresponds to crack propagation. The crack in the aluminum material can produce a strain concentration or gradient zone, which contains complex strain patterns. If 

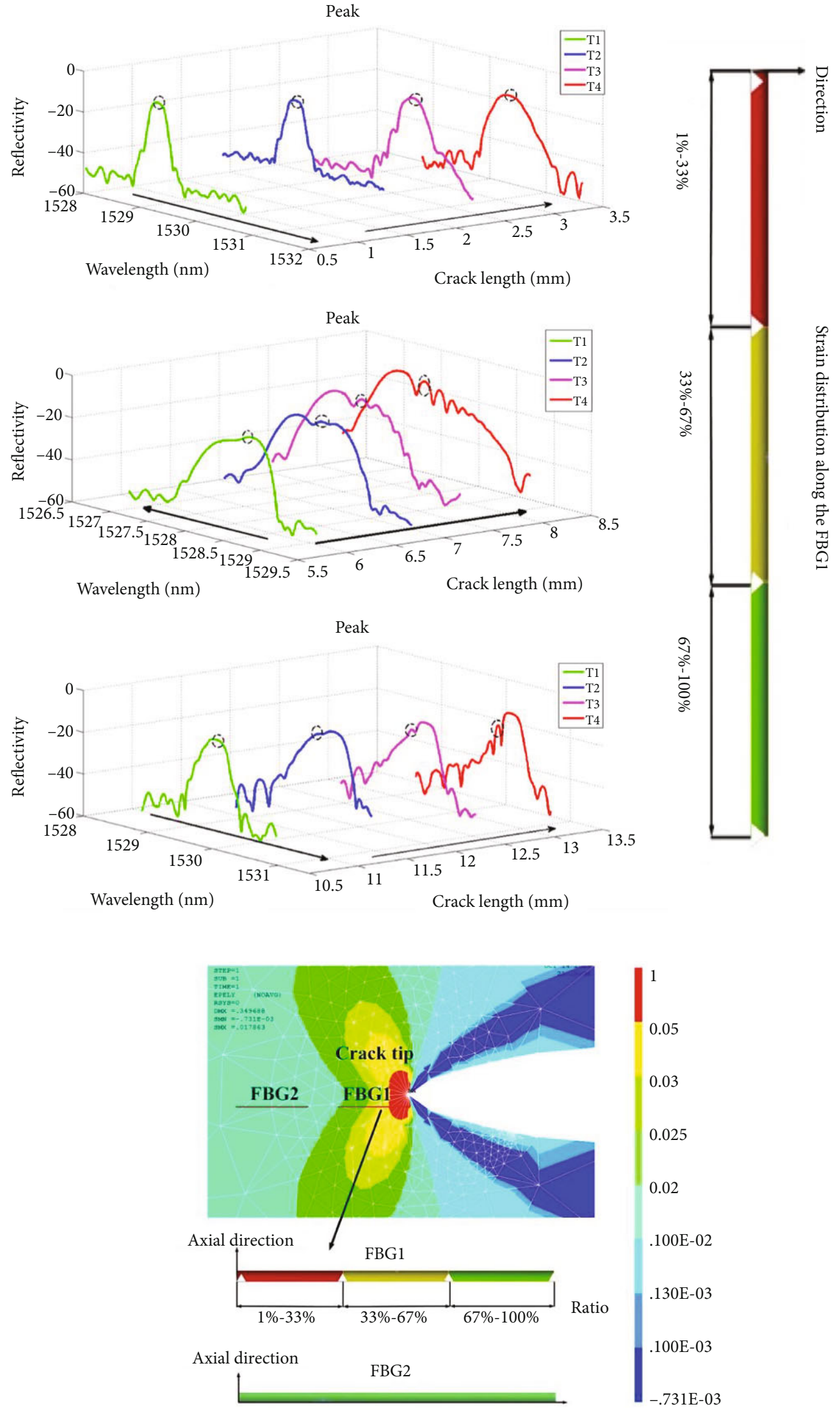

(a) Spectra of FBG1

Figure 8: Continued. 


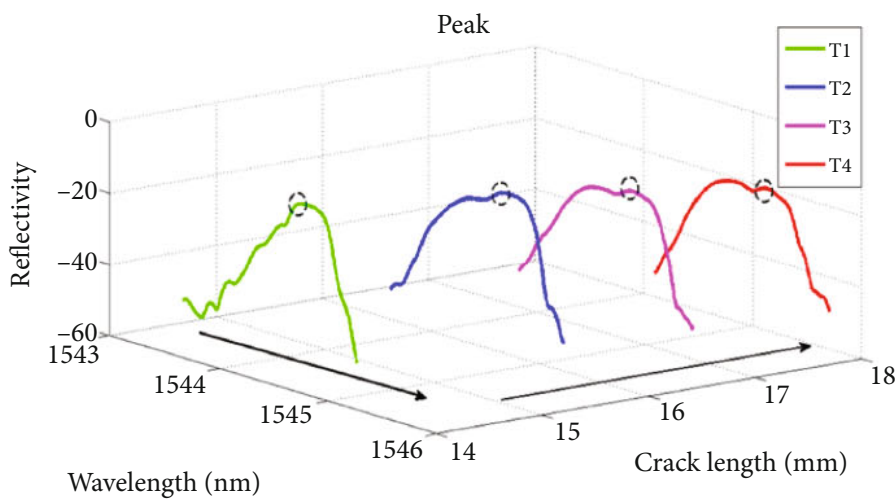

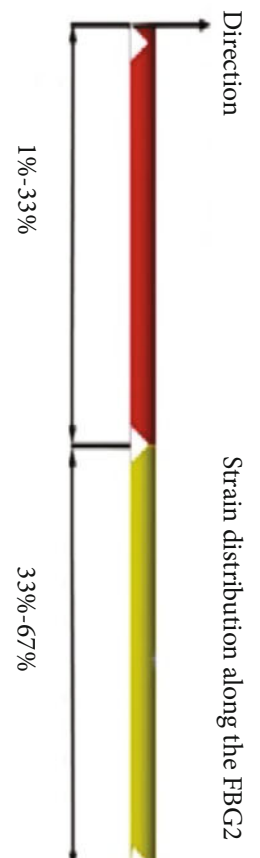
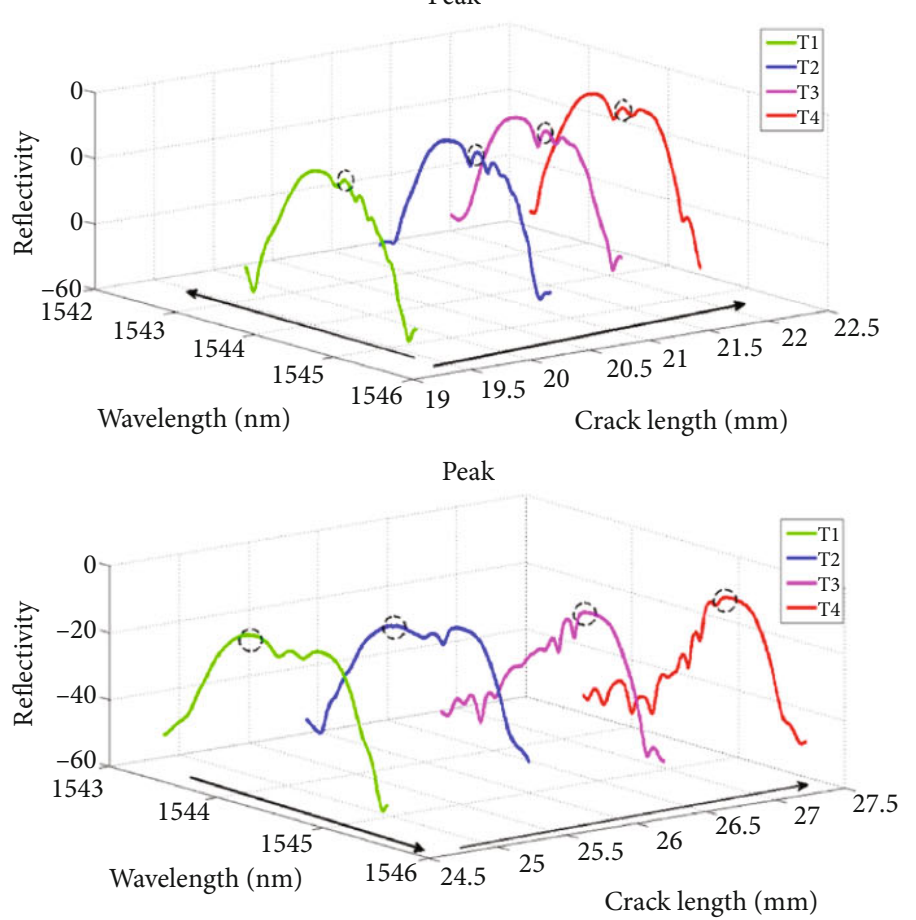

웡

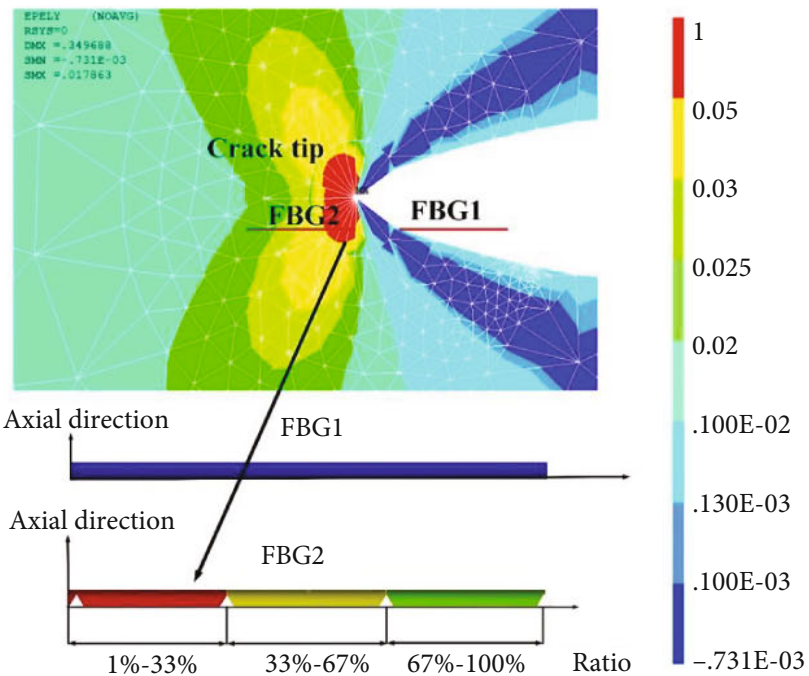

(b) Spectrum of FBG2

FIgURE 8: The corresponding spectra for different crack lengths. 


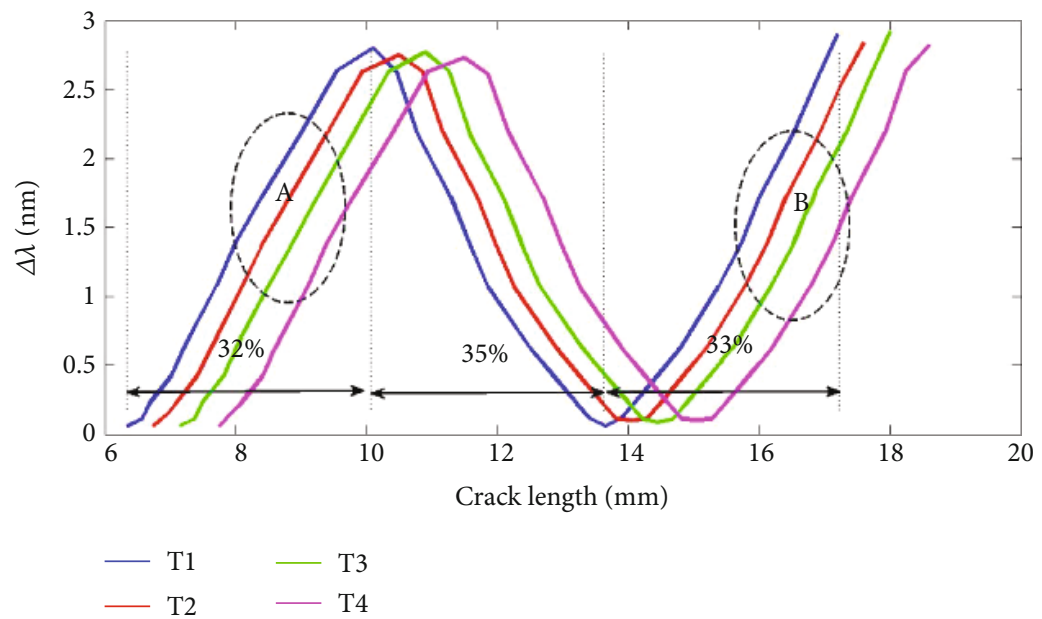

FIGURE 9: The primary wavelength variation vs. crack length.

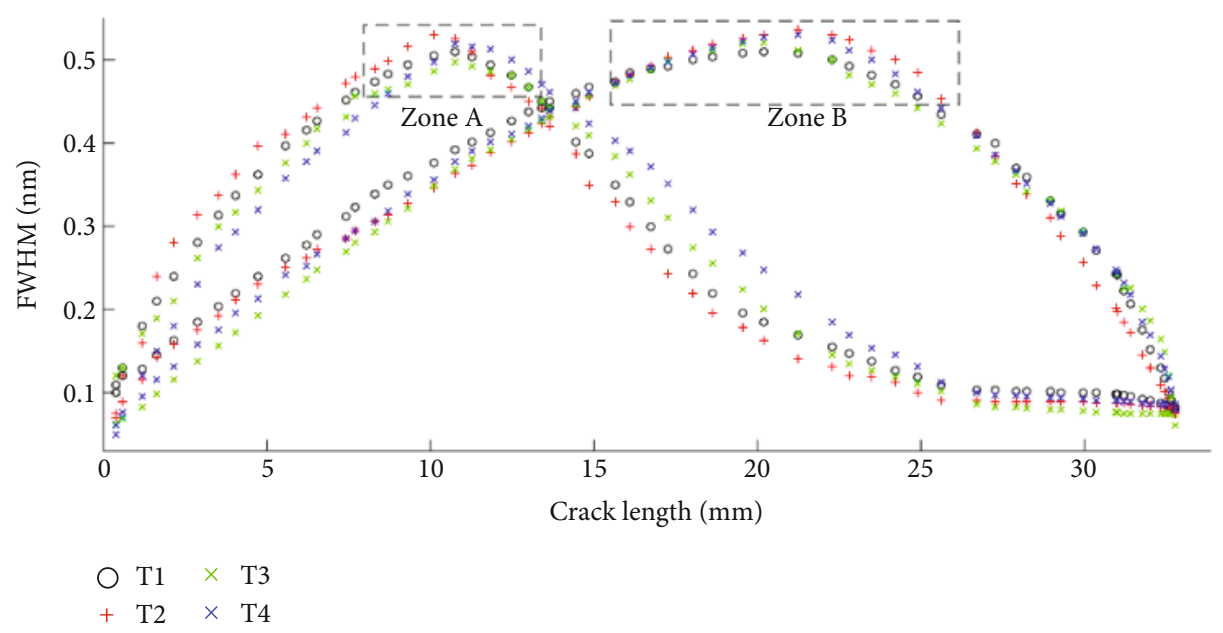

Figure 10: FWHM vs. crack length data for all four parallel plates.

different strain patterns are applied on the grating, the grating will suffer with the same nonuniform strain distribution pattern, causing a sensor response that is significantly more complicated relative to a uniform case [26]. The nonuniform strain along the grating will change the periodicity of the grating pattern. Hence, the grating pattern is modified from a uniform to a chirped configuration [27], as shown in Figure 10.

3.3.3. Number of Subordinate Peaks. The crack in the aluminum material can produce a strain concentration, which contains complex strain patterns. The spectrum distortion corresponds to the strain pattern in the grating. Meanwhile, if the grating suffers with a similar nonuniform strain pattern, the sensor response is significantly more complicated compared to a uniform case [18]. This means that the spectral distortion relates to both the ratio of crack tip lying in the whole grating length and the location of the crack tip in the grating. The number of subordinate peaks is dependent on this ratio.
In this research, spectral datasets are collected by fixing four parallel specimens under cyclic fatigue tests. The reflected spectral data were normalized as for the spectral subordinate peak number method. Figure 11 displays a schematic diagram of the subordinate peak number counting. Although the high-order peaks had been filtered using the FBG sensing demodulation SM 125, the higher-order peaks imply no significant contribution to the crack propagation. Thus, the intensity of the reflection spectrum was normalized by the intensity of the highest component. The reflected spectrum data was selected with $\bar{r} \geq 0.5$ (i.e., full width at half maximum).

If the strain field is changing over a short effective segment of the whole grating length, the cubic strain pattern as the main form distributed along the grating. As only a discrete number of peaks are calculated, the relationship is not as smooth as the spectral primary wavelength measure.

At the $1 \%-67 \%$ ratio of the crack length lying in the whole grating, the number of the subordinate peaks shows an upward trend. This is especially important during the 


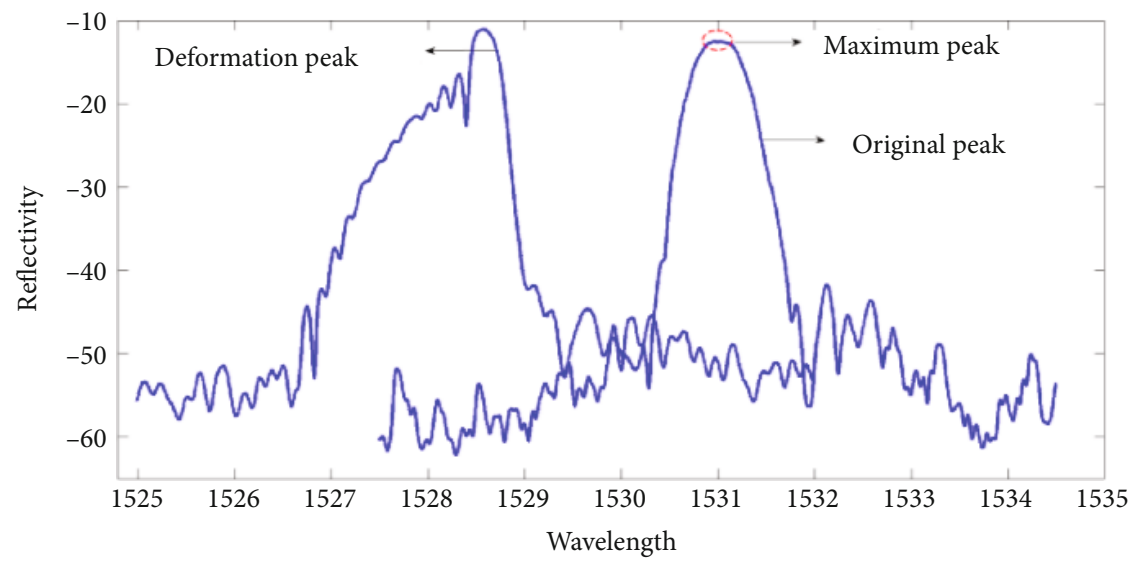

(a) Primary peak seeking

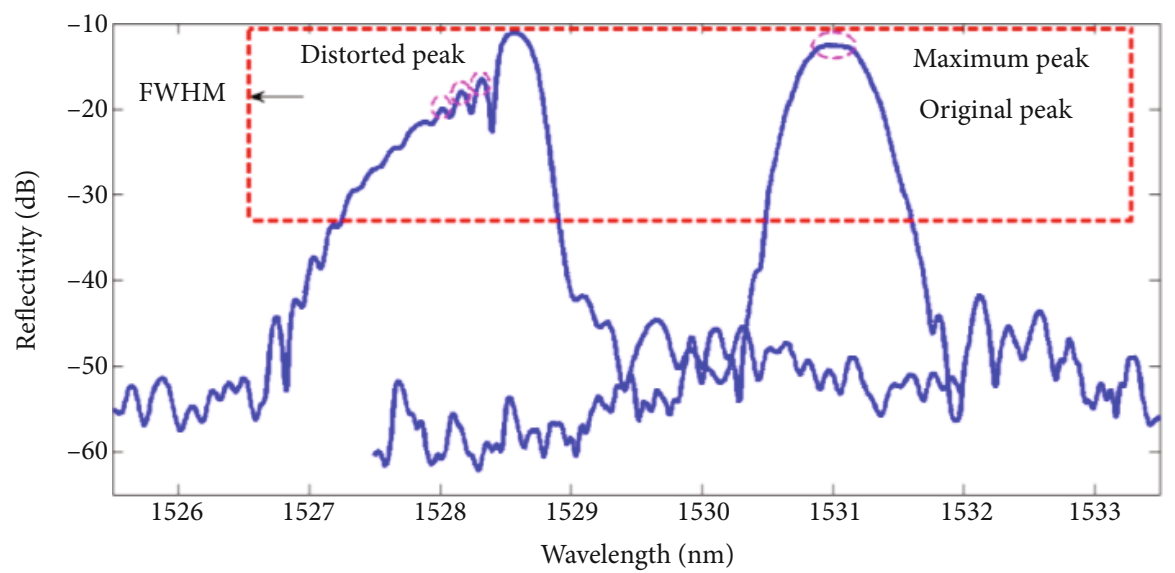

(b) Threshold determination

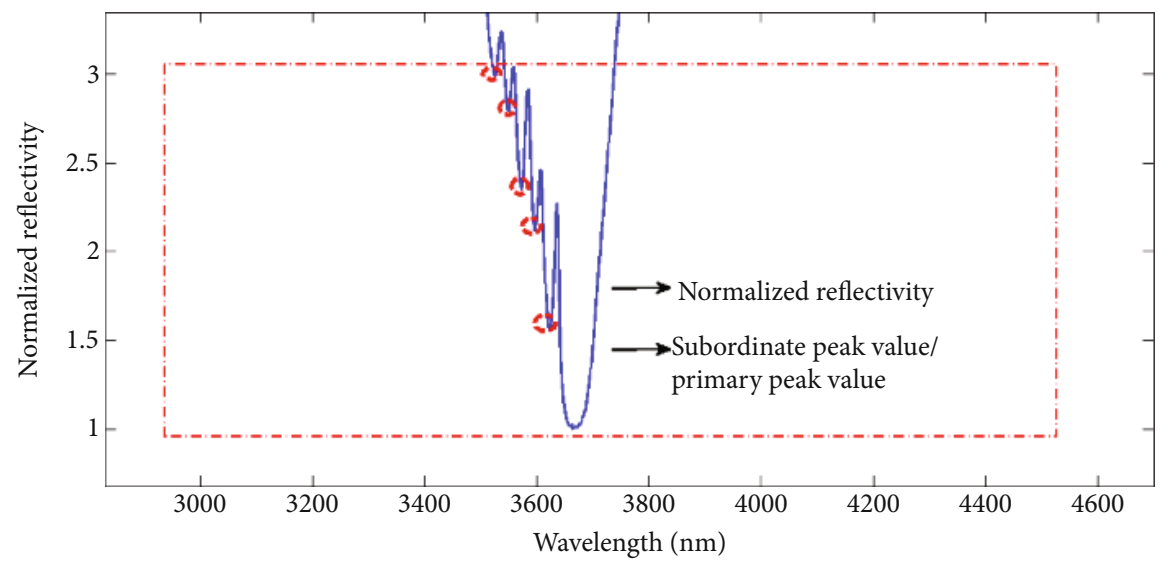

(c) Nominalized reflectivity

FIGURE 11: The schematic diagram of the subordinate peak number counting.

ratio of $1 \%-32 \%$, where the subordinate peak number increased rapidly. The experimental spectral bandwidth is plotted for four parallel plates in Figure 12.

Thus, the number of subordinate peaks may be useful as an indicator of imminent failure of the aluminum material.

3.3.4. The Subordinate Wavelength Skewing. The location of subordinate peaks corresponds to the location of strain concentration on the FBG sensors. At the initial stage, about
$15 \%$ ratio of crack length lying in the whole grating causes slitting of the spectra and distortion of the spectral shape. The subordinate peak appears at the shorter wavelength, and the wavelength only increased due to the strain value concentered on the short effective sensitive grating. Meanwhile, at about $32 \%$ ratio of the crack length lying in the whole grating, a similar trend of subordinate peak wavelength can be observed for crack propagation. As our previous research shows, this depends on the strain pattern 


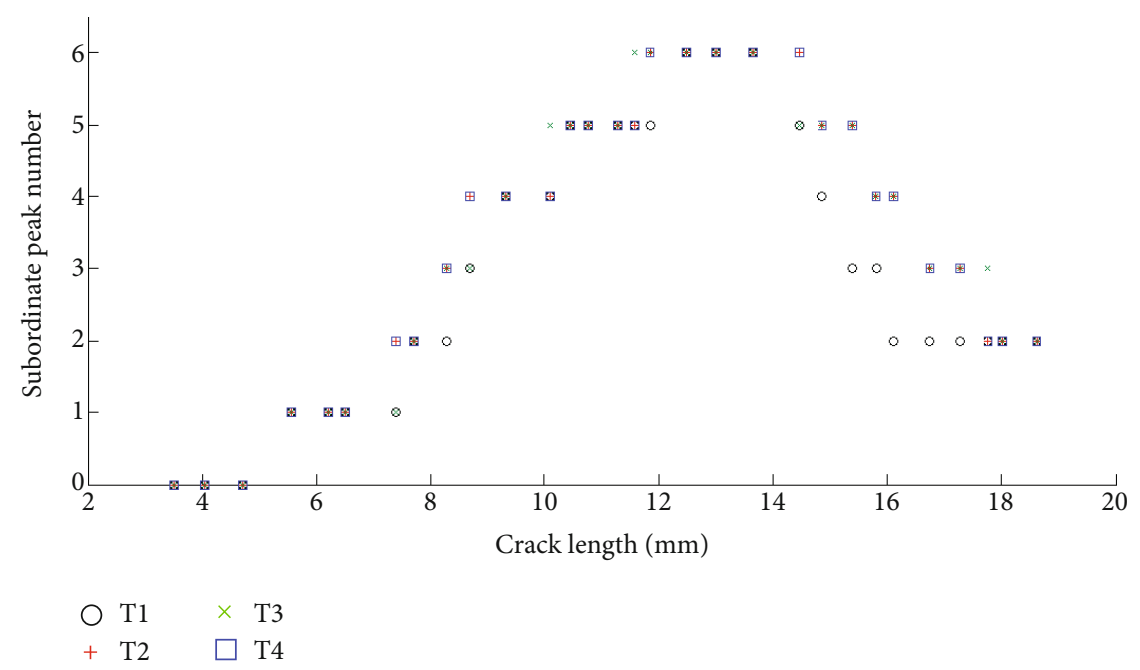

(a) FBG1

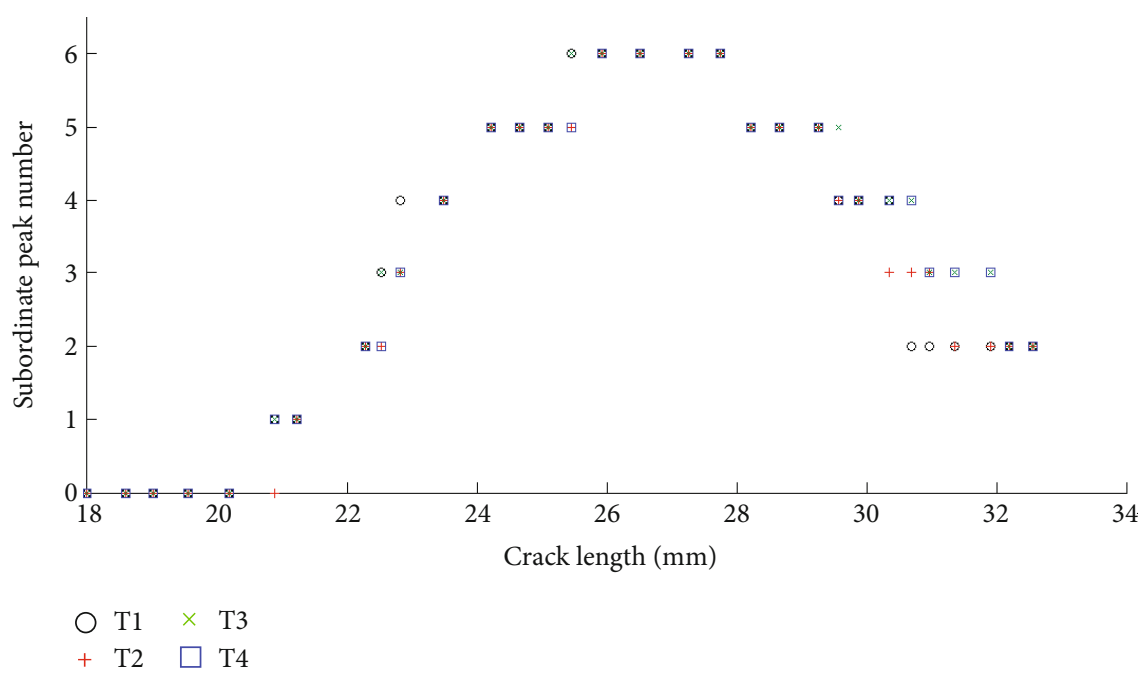

(b) FBG2

FIgURE 12: Number of subordinate peaks vs. crack length.

distribution along the grating. It must also include the different shapes of the strain distributions, for the analysis directly reflected the shape skewing of the subordinate peak.

Afterwards, the spectra become enlarged, and the subordinate peak skews at a longer wavelength and drifts to the same orientation. The nonuniform strain along the grating will change the periodicity of the grating pattern. In this way, the grating pattern is modified from a uniform to a chirped configuration [28]. Meanwhile, the subordinate peak wavelength shows a significant decline at the strain pattern transition region with the average strain decrease, as shown in zone $\mathrm{A}$ in Figure 13. Nevertheless, as the crack length increases, the strain pattern is distributed along the variable grating, and the widths of the measured spectra increased.

It is confirmed that oscillations of the spectrum can be related to the nonuniform strain distribution along the grating caused by cracks. Thus, they serve nonuniform strain patterns along the grating, and it is assumed to be the principal reason for the spectral oscillation [29]. Meanwhile, when the ratio reaches to about $67 \%$, the subordinate peak appears at a shorter wavelength, and the strain value distribution on the effective sensing region increased. Thus, the subordinate peak wavelength increased rapidly, as shown in zone B in Figure 13. The reflection spectra were even slit into several peaks at the ultimate break of the grating, at about $15 \%$ ratio of crack length lying in the whole grating.

To further explore the subordinate wavelength variation, the experimental data of four samples tested were analyzed, as shown in Figure 13.

\section{Discussion}

4.1. Subordinate Wavelength Skewing. When the compression strain is identified by the grating, the spectrum moves to a shorter wavelength. In contrast, when the tensile strain is detected by the grating, the spectrum moves to a longer wavelength [30]. The strain distribution around the crack tip is highly variable. It is worth mentioning that if the FBG was submitted to different strain patterns along the grating, different lower peaks will distort the reflected peak. 


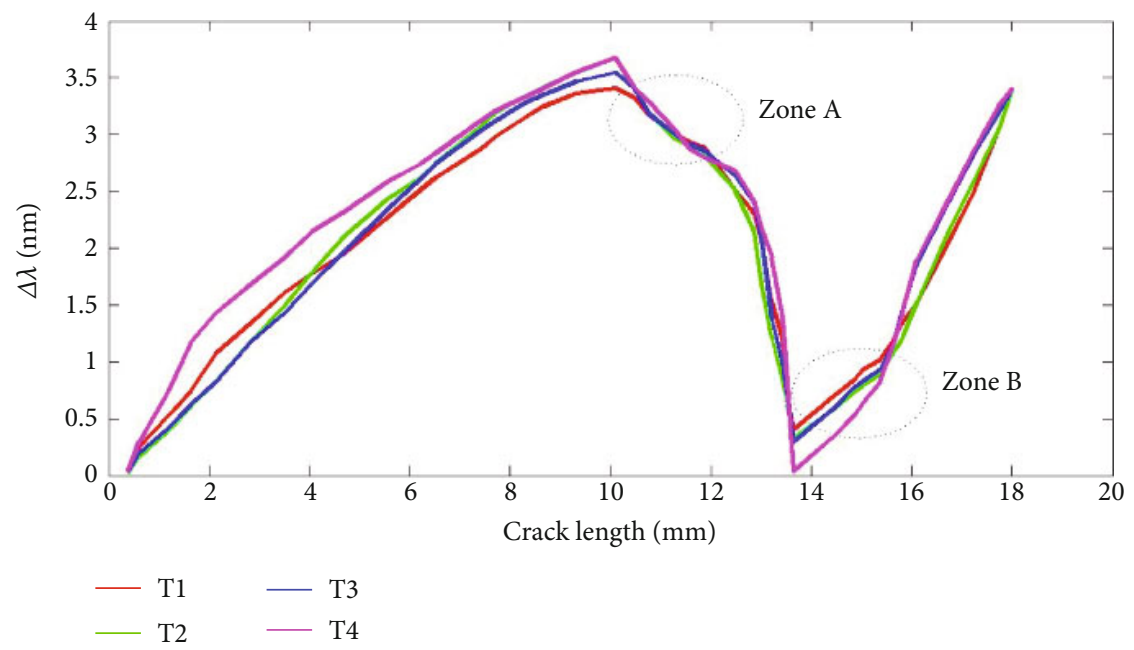

(a) FBG1

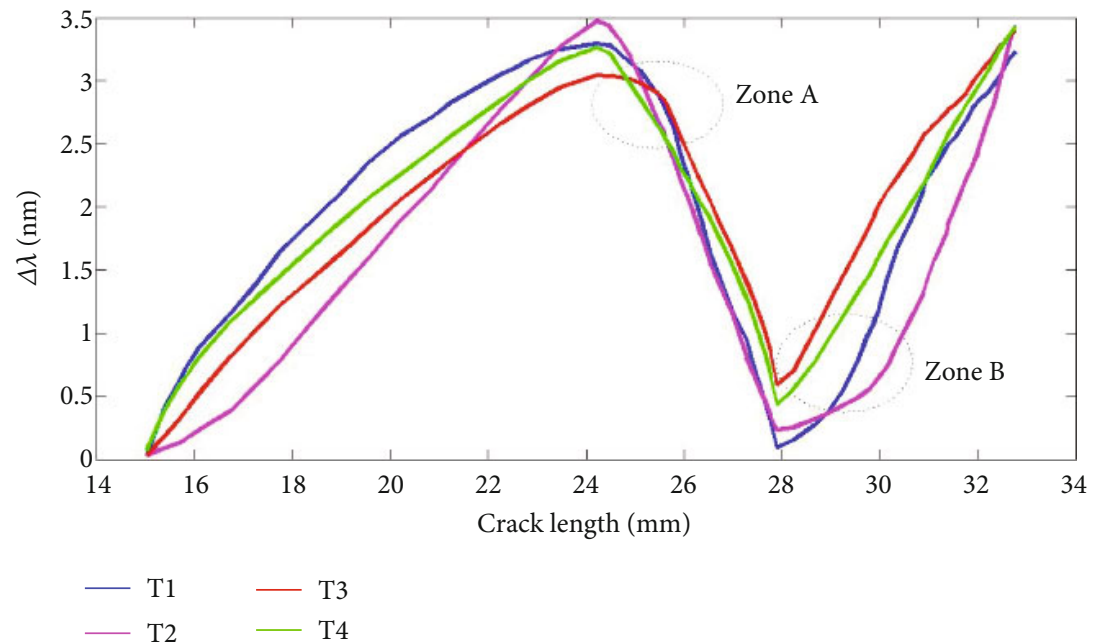

(b) FBG2

FIGURE 13: Subordinate peak skewing vs. crack length.

The slitting of the spectra occurred and the spectral shape becomes distorted. Meanwhile, the primary wavelength, FWHM, subordinate peak number, and subordinate wavelength skewing with the strain pattern change along the grating.

It is assumed that the subordinate peak location skewing corresponds to the change of the strain pattern along the grating, with respect to the crack propagation. This is especially true at the strain pattern transition region, which is located at the ratio $32-34 \%$ of crack length lying in the whole grating.

4.2. Spectral Distortion. Literature [31] shows that both elastic and plastic strains can be experienced in the grating. In combination with complex strain distributions around the crack tip, a serve strain distribution pattern could be sensed by the grating, such as linear, quadratic, and cubic strain distributions. The literature further reveals that the linear, quadratic, and cubic strain patterns along the grating determine the subordinate peak location.
Thus, it is assumed that the complex strain pattern perceived by the grating could be the chief reason of spectrum distortion, correlating with the ratio of crack length lying in the whole grating.

\section{Conclusion}

To determine the characteristic damage variation of FBG sensors with the crack propagation, FBG shows significant changes in the strain due to damage in the specimen. The four characteristic parameters were abstracted by analyzing experimental data. This was followed by the correlation of damage characteristics with crack length and investigated with the four damage characteristics, namely, the primary wavelength, the subordinate peak number, the FWHM, and the subordinate wavelength skewing.

During crack propagation, the subordinate peak skewed with the strain pattern transition region, with the ratio 32$34 \%$ of crack length lying in the grating length. Meanwhile, the FWHM, the primary wavelength, and the subordinate 
peak number correspond to the subordinate peak skewing. Thus, it is confirmed that subordinate peak skewing can be applied as an effective indicator to evaluate the crack propagation for the aluminum.

Furthermore, the FBG-based demodulation method can be used as an interesting and alternative technique for realtime detection of transverse cracks in aluminum.

\section{Data Availability}

The processed data required to reproduce these findings cannot be shared at this time as the data also forms part of an ongoing study.

\section{Conflicts of Interest}

The authors declare no competing interests.

\section{Authors' Contributions}

Bo Jin and Cunbo Lu conceived the key idea and designed the experiments. Feng Zhang provided the academic support and checked the manuscript. Weifang Zhang performed the experiment and contributed to optical measurement tools. All authors made contributions to writing and revising the manuscript.

\section{References}

[1] G. C. Kahandawa, J. Epaarachchi, H. Wang, and K. T. Lau, "Use of FBG sensors for SHM in aerospace structures," Photonic Sensors, vol. 2, no. 3, pp. 203-214, 2012.

[2] J. P. Ou, "Some recent advance of intelligent monitoring system for civil infrastructures in mainland China," in Proceedings of the First International Conference on Structural Health Monitoring and Intelligent Infrastructures, pp. 131144, Tokyo, Japan, 2003.

[3] D. Kinet, P. Mégret, K. W. Goossen, L. Qiu, D. Heider, and C. Caucheteur, "Fiber Bragg grating sensors toward structural health monitoring in composite materials: challenges and solutions," Sensors, vol. 14, no. 4, pp. 7394-7419, 2014.

[4] N. Takeda, Y. Okabe, and T. Mizutani, "Damage detection in composites using optical fibre sensors," Proceedings of the Institution of Mechanical Engineers Part G Journal of Aerospace Engineering, vol. 221, no. 4, pp. 497-508, 2007.

[5] P. Childs, A. C. L. Wong, W. Terry, and G. D. Peng, "Measurement of crack formation in concrete using embedded optical fibre sensors and differential strain analysis," Measurement Science and Technology, vol. 19, no. 6, article 065301, 2008.

[6] Sante and R. Di, "Fibre optic sensors for structural health monitoring of aircraft composite structures: recent advances and applications," Sensors, vol. 15, no. 8, pp. 18666-18713, 2015.

[7] R. Ramly, W. Kuntjoro, and M. K. Abd Rahman, "Embedded FBG sensor in aircraft smart composite materials for structural monitoring," Applied Mechanics \& Materials, vol. 393, no. 393, pp. 311-316, 2013.

[8] A. Rajabzadeh, R. M. Groves, R. C. Hendriks, and R. Heusdens, "Modelling non-uniform strain distributions in aerospace composites using fibre Bragg gratings," in 25th
International Conference on Optical Fiber Sensors, Jeju, Korea, 2017.

[9] R. Correia, E. Chehura, S. W. James, and R. P. Tatam, “A pressure sensor based upon the transverse loading of a sub-section of an optical fibre Bragg grating," Measurement Science of Technology, vol. 18, no. 10, pp. 3103-3110, 2007.

[10] M. A. Soto, J. A. Ramírez, and L. Thévenaz, "Optimizing image denoising for long-range Brillouin distributed fiber sensing," Journal of Lightwave Technology, vol. 36, no. 4, pp. 1168$1177,2018$.

[11] M. Li and J. Yao, "All-fiber temporal photonic fractional Hilbert transformer based on a directly designed fiber Bragg grating," Optics Letters, vol. 35, no. 2, p. 223, 2010.

[12] Y. Chen, X. Yang, H. L. Liu, K. Yang, and Y. L. Zhang, "Processing FBG sensing signals with exponent modified Gaussian curve fitting peak detection method," Spectroscopy and Spectral Analysis, vol. 36, no. 5, pp. 1526-1531, 2016.

[13] S. Jiao, W. Shi, and L. Qian, "Self-adaptive partial discharge denoising based on variational mode decomposition and wavelet packet transform," in 2017 Chinese Automation Congress (CAC), Jinan, China, 2017.

[14] M. Ramakrishnan, G. Rajan, Y. Semenova, and G. Farrell, "Overview of fiber optic sensor technologies for strain/temperature sensing applications in composite materials," Sensors, vol. 16, no. 1, 2016.

[15] B. Jin, W. Zhang, F. Ren, M. Zhang, W. Dai, and Y. Wang, "Mechanism of subordinate peak skewing of FBG sensor during cracks propagation monitoring on aluminum alloy structure," Journal of Sensors, vol. 2017, Article ID 2857693, 7 pages, 2017.

[16] L. J. Cai, Z. Y. Li, Z. H. Tang, W. Meng, and Q. Liu, "A novel peak-seek algorithm used in FBG sensor demodulation system for vibration monitoring," Applied Mechanics \& Materials, vol. 160, pp. 135-139, 2012.

[17] C. Fernando, A. Bernier, S. Banerjee, G. G. Kahandawa, and J. Eppaarchchi, "An investigation of the use of embedded FBG sensors to measure temperature and strain inside a concrete beam during the curing period and strain measurements under operational loading," Procedia Engineering, vol. 188, pp. 393-399, 2017.

[18] M. Kreuzer, "Strain measurement with fiber Bragg grating sensors," HBM, 2006.

[19] S. Takeda, Y. Okabe, and N. Takeda, "Monitoring of delamination growth in CFRP laminates using chirped FBG sensors," Journal of Intelligent Material Systems and Structures, vol. 19, no. 4, pp. 437-444, 2008.

[20] M. Ussorio, H. Wang, S. L. Ogin et al., "Modifications to FBG sensor spectra due to matrix cracking in a GFRP composite," Construction and Building Materials, vol. 20, no. 1-2, pp. 111-118, 2006.

[21] N. Takeda, Y. Okabe, J. Kuwahara, S. Kojima, and T. Ogisu, "Development of smart composite structures with small-diameter fiber Bragg grating sensors for damage detection: quantitative evaluation of delamination length in CFRP laminates using lamb wave sensing," Composites Science and Technology, vol. 65, no. 15-16, pp. 25752587, 2005.

[22] B. Jin, W. Zhang, M. Zhang, F. Ren, W. Dai, and Y. Wang, "Investigation on characteristic variation of the FBG spectrum with crack propagation in aluminum plate structures," Materials, vol. 10, no. 6, p. 588, 2017. 
[23] K. Peters, P. Pattis, J. Botsis, and P. Giaccari, "Experimental verification of response of embedded optical fiber Bragg grating sensors in non-homogeneous strain fields," Optics and Lasers in Engineering, vol. 33, no. 2, pp. 107-119, 2000.

[24] G. Guo, D. A. Hackney, M. Pankow, and K. Peters, "Interrogation of a spectral profile division multiplexed FBG sensor network using a modified particle swarm optimization method," Measurement Science \& Technology, vol. 28, no. 5, Article ID 055204, 2017.

[25] K. Peters, M. Studer, J. Botsis, A. Iocco, H. Limberger, and R. Salathé, "Embedded optical fiber Bragg grating sensor in a non-uniform strain field: measurements and simulations," Experimental Mechanics, vol. 41, no. 1, pp. 19-28, 2001.

[26] Y. Okabe, S. Yashiro, T. Kosaka, and N. Takeda, "Detection of transverse cracks in CFRP composites using embedded fiber Bragg grating sensors," Smart Materials \& Structures, vol. 9, no. 6, pp. 832-838, 2000.

[27] K. J. Peters, M. Studer, J. Botsis, A. Iocco, H. G. Limberger, and R.-P. Salathe, "Measurement of stress concentrations using embedded optical fiber Bragg grating sensors," in 1999 Symposium on Smart Structures and Materials. International Society for Optics and Photonics, Newport Beach, CA, United States, 1999.

[28] A. Othonos, "Fiber bragg gratings," The Review of Scientific Instruments, vol. 68, no. 12, pp. 4309-4341, 1997.

[29] S. Cięszczyk and P. Kisała, "Inverse problem of determining periodic surface profile oscillation defects of steel materials with a fiber Bragg grating sensor," Applied Optics, vol. 55, no. 6 , pp. 1412-1420, 2016.

[30] J. F. Botero-Cadavid, J. D. Causado-Buelvas, and P. Torres, "Spectral properties of locally pressed fiber Bragg gratings written in polarization maintaining fibers," American Institute of Physics, 2008.

[31] J. Zhou, Z. Zhou, and D. Zhang, "Study on strain transfer characteristics of fiber Bragg grating sensors," Journal of Intelligent Material Systems and Structures, vol. 21, no. 11, pp. 11171122, 2010. 